\title{
Analysis of Acoustic Wave Propagation in a Power Transformer Model
}

\author{
D. Wotzka, T. Boczar And D. ZMARZLY* \\ Faculty of Electrical Engineering, Automatic Control and Computer Science \\ Opole University of Technology, Mikołajczyka 5, 45-271 Opole, Poland
}

\begin{abstract}
Analyses of acoustic wave propagation in a model power transformer are presented in the paper. The acoustic wave is induced by partial discharges that are simulated at predefined coordinates in the core and winding. Propagation of the numerical calculated acoustic wave is analyzed within the transient state. Achieved results indicate that the space and time distributions of the acoustic pressure depend on the induction position. Furthermore, a greater pressure gradient is observed in domains with higher speed of sound while the largest amplitude occurs at the vicinity of the induction position.
\end{abstract}

PACS numbers: 52.80.-s, 43.28.Js

\section{Introduction}

One of the common reasons for power transformer degradation is presence and activation of partial discharges. Measurements of the acoustic partial discharge (PD) in power transformers are focused on PDs location estimation, e.g. by use of acoustic emission sensors $[1,2]$. For this reason it is helpful and necessary that the propagation of the acoustic wave created by the PD is known. Because there are found only a few publications, which consider analysis of acoustic waves in power transformers [3-14], it was recapitulated that the analysis of the acoustic wave propagation in power transformers seems to be lacking; therefore, this was investigated in this work.

Partial discharges are simulated at two different coordinates in the core and in the winding for study the acoustic wave, which propagates through the transformer. A 3D model of a typical $1 \mathrm{MV}$ A oil immersed power transformer has been developed using the Pressure Acoustic mode of the COMSOL Multiphysics software. The acoustic pressure is solved numerically using the finite element method supported by COMSOL within the transient state. Analysis of achieved results has shown that the acoustic pressure amplitude depends on distance between source and observation position.

\section{Mathematical model definition}

The acoustic pressure $L$ is measured in decibels $[\mathrm{dB}]$ and is given by Eq. (1) [9]:

\footnotetext{
* corresponding author; e-mail: dzmarzly@gmail.com
}

$$
L=20 \log \left(p / p_{0}\right),
$$

where $p$ - the effective value of the acoustic pressure in pascal [Pa], $p_{0}$ - reference pressure equal to $20 \mu \mathrm{Pa}$ (the reference pressure is the acoustic pressure by which the sound becomes to be hearable). The acoustic pressure $p$ $[\mathrm{Pa}]$ in lossless media is numerically calculated by Eq. (2):

$$
\nabla \cdot\left(\frac{-1}{\rho_{0}} \nabla p\right)=\frac{\partial Q}{\partial t}+\frac{1}{c^{2} \rho_{0}} \frac{\partial^{2} p}{\partial t^{2}}
$$

where $\rho_{0}\left[\mathrm{~kg} / \mathrm{m}^{3}\right]$ - the fluid/solid density, $c[\mathrm{~m} / \mathrm{s}]$ - the speed of sound, $Q\left[\mathrm{~m}^{3} / \mathrm{s}\right]$ - the monopole source of energy flow that identifies in this work the simulated partial discharge. $Q$ is time dependent as shown in Eq. (3):

$$
Q(t)=\frac{1}{1+\mathrm{e}^{-a(t-b)}} \mathrm{e}^{-(t-b) f} \cos (2 \pi f(t-b)),
$$

where $a$ - the dimensionless impulse wide factor equal to $1 \times 10^{8}, b$ - time offset equal to $3 \mu \mathrm{s}, f$ - the frequency equal to $1 \mathrm{MHz}$. Figure 1 shows time run of $Q$ (energy of the simulated PD).

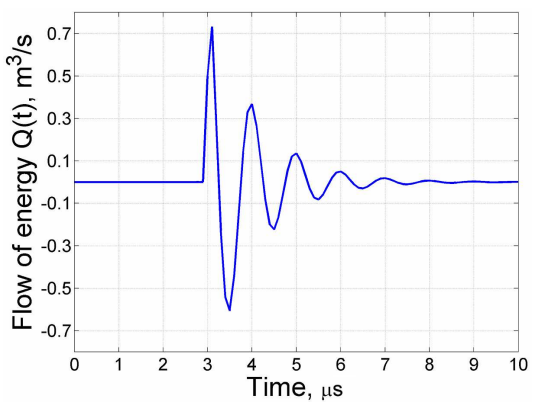

Fig. 1. Time run of the simulated PD.

The geometry of the model power transformer is presented in Fig. 2. The transformer is modeled as a 
$2.6 \times 1 \times 1.5 \mathrm{~m}^{3}$ box that is filled with transformer oil. Three cores, surrounded by windings, are assumed to be cylindrical objects. Cores and windings are mounted with two bases, at the top and at the bottom respectively, in the central part of the transformer tank. The simulated material properties of transformer elements are summarized in Table.

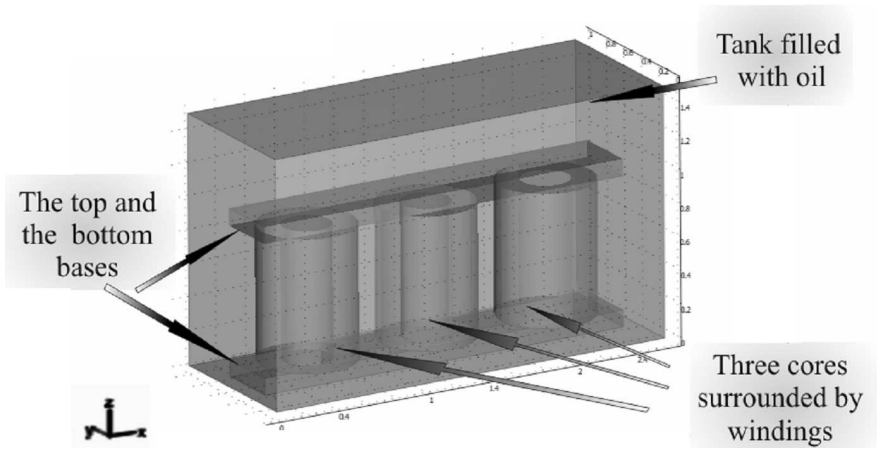

Fig. 2. The geometry of the modeled power transformer.

TABLE

Material properties of modeled transformer elements.

\begin{tabular}{c|c|c|c}
\hline \hline Part name & Material & $\begin{array}{c}\text { Density } \\
{\left[\mathrm{kg} / \mathrm{m}^{3}\right]}\end{array}$ & $\begin{array}{c}\text { Speed of sound } \\
{[\mathrm{m} / \mathrm{s}]}\end{array}$ \\
\hline core and bases & structural steel & 7850 & 5100 \\
windings & copper & 8700 & 4760 \\
oil & transformer oil & 890 & 1390
\end{tabular}

The applied mathematical model does not take into account any fluid dynamics or other than acoustical physical issues; thus: oil flow, heat transfer and electromagnetic field coupling are not considered. Furthermore, the medium is assumed to be lossless so the acoustic wave attenuates neither in the core, windings nor in the oil. The transformer outer walls are specified as "perfectly soft" and satisfy Eq. (4):

$$
p=0 \text {, }
$$

where $p$ - the acoustic pressure.

At the interface between the oil, windings and core the continuity boundary is modeled

$$
n\left[\left(\nabla p / \rho_{0}\right)_{1}-\left(-\nabla p / \rho_{0}\right)_{2}\right]=0,
$$

where $n$ - the outward oriented normal vector, $\rho_{0}$ - the fluid/solid density, $p$ - the acoustic pressure; indices 1 and 2 state for the first and the second medium.

The GMRES linear system solver and the geometric multigrid preconditioner have been applied for the transient analysis with 100 time steps each of 100 ns. The relative and absolute step tolerances were set to $10 \mathrm{~ms}$ and $1 \mu$ s respectively; other settings were set to default. The mesh consisted of 6986 tetrahedral elements resulting with 28837 unknowns. The minimum element quality was 0.2154 . The model simulation was run on a Genuine
Intel@ Core $^{\mathrm{TM}}$ Duo T2500, $1.99 \mathrm{GHz}, 2$ GB RAM computer. COMSOL used 474 byte of memory and needed 1365 seconds ( $22 \mathrm{~min}$ and $45 \mathrm{~s}$ ) of CPU time for solution of the second order finite element model.

\section{Results and discussion}

Partial discharges have been simulated at two different Cartesian coordinates: first, in the middle of the left core at point $S=(0.5,0.5,0.5)[\mathrm{m}]$, second, in the left winding at point $W=(0.7,0.5,0.5)[\mathrm{m}]$. The acoustic pressure has been estimated and analyzed within the transient state. The space distribution of the acoustic pressure $L$ expressed in decibels in the first $5 \mu$ s following the $\mathrm{PD}$ explosion in the core (at point $S$ ) is shown in Figs. 3 to 5 .

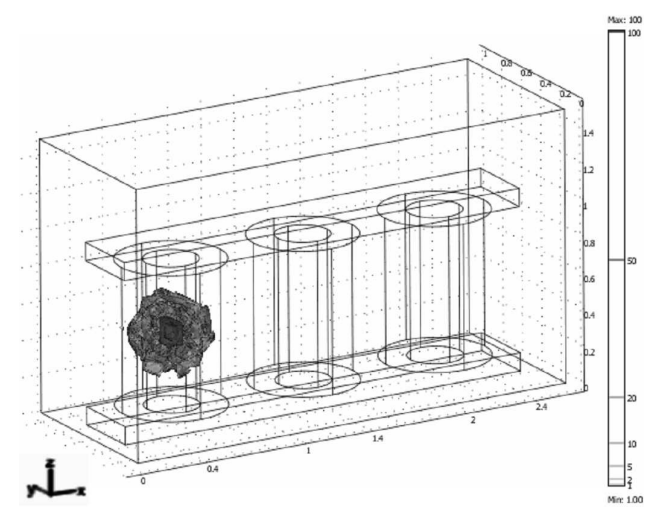

Fig. 3. Space distribution of the acoustic pressure $L$, $\mathrm{dB} 0.2 \mu$ s after the PD explosion time.

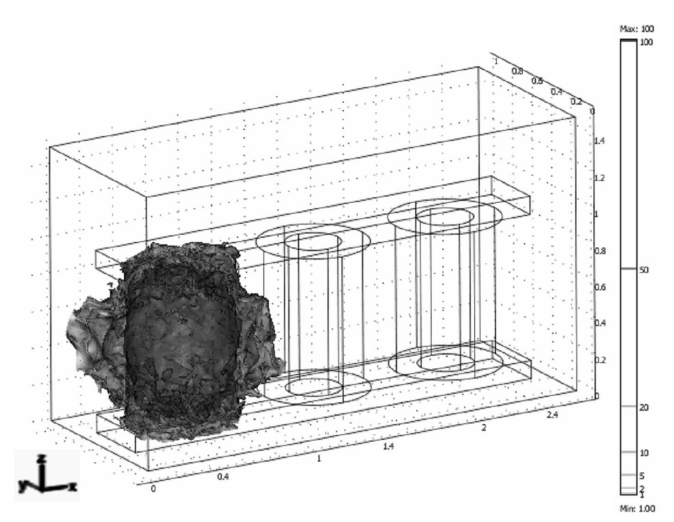

Fig. 4. Space distribution of the acoustic pressure $L$, $\mathrm{dB} 0.3 \mu$ s after the PD explosion time.

The isobars indicate rapid pressure gradient in the first microsecond following the PD start. There are no further significant changes in the pressure space distribution after the first microsecond after the PD.

Figures 6 and 7 show the time distribution of the pressure $L, \mathrm{~dB}$ at the corners of the transformer tank at position $x=1 \mathrm{~m}$. The PD was induced inside the core 


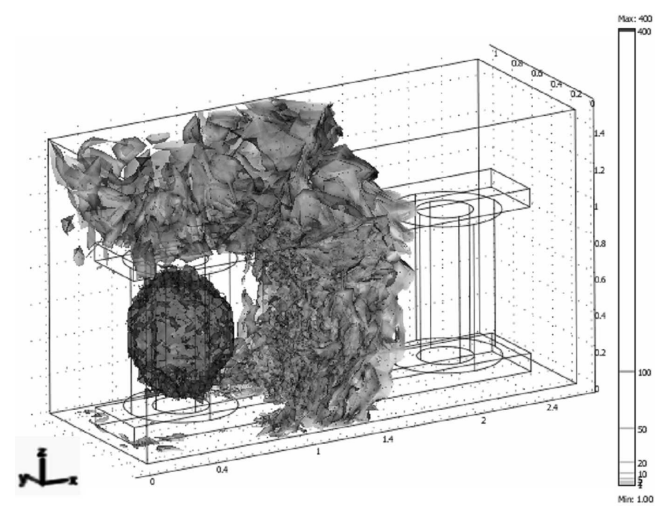

Fig. 5. Space distribution of the acoustic pressure $L$, dB $5 \mu$ s after the PD explosion time.

and in the winding, respectively. Relative high values of $L$ around $300 \mathrm{~dB}$ indicate large noise inside the transformer tank.

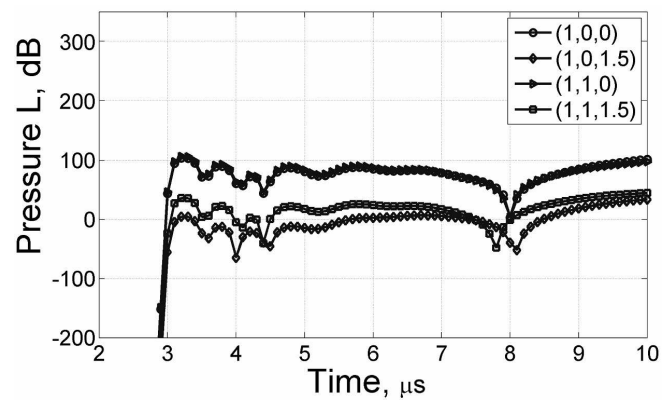

Fig. 6. Time distribution of the acoustic pressure $L$ at specified Cartesian coordinates $x, y, z$ (see legend). The $\mathrm{PD}$ was induced in the core.

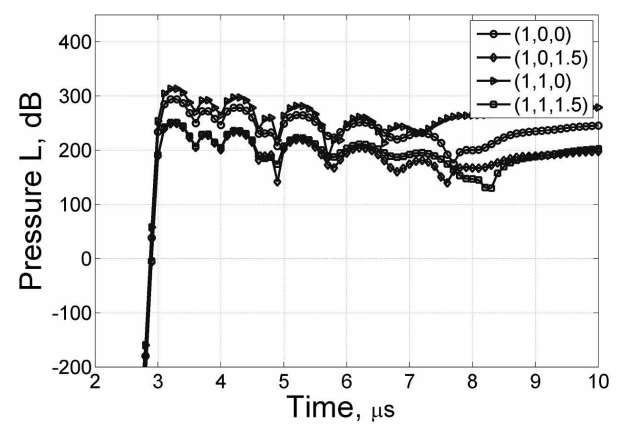

Fig. 7. Time distribution of the acoustic pressure $L$ at specified Cartesian coordinates $x, y, z$ (see legend). The $\mathrm{PD}$ was induced in the winding.

Figures 8 and 9 show the time distribution of the pressure $L, \mathrm{~dB}$ at the corners of the transformer tank at position $x=2.6 \mathrm{~m}$. The PD was induced inside the core and in the winding, respectively.

Regarding Figs. 6 to 9 one can indicate that in the first $1 \mu$ s after the PD explosion pressure $L$ distributes quite

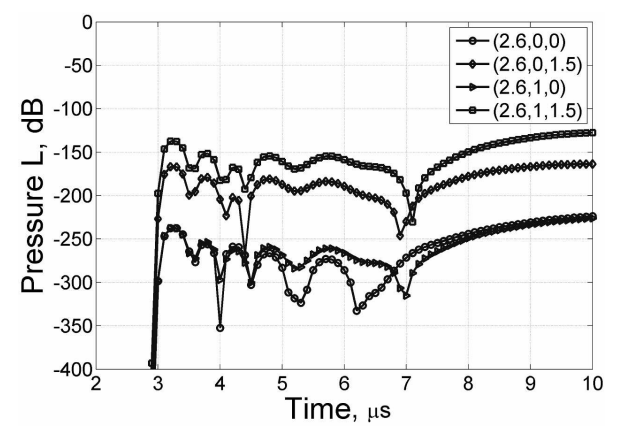

Fig. 8. Time distribution of the acoustic pressure $L$ at specified Cartesian coordinates $x, y, z$ (see legend) as the $\mathrm{PD}$ induced in the core.

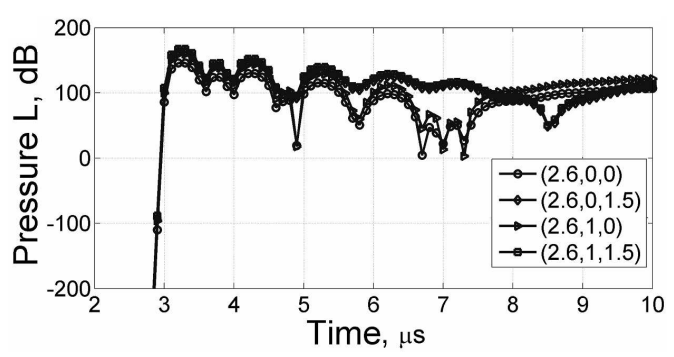

Fig. 9. Time distribution of the acoustic pressure $L$ at specified Cartesian coordinates $x, y, z$ (see legend) as the $\mathrm{PD}$ induced in the winding.

similar in all cases; after this time $(t>4 \mu \mathrm{s})$, curves differ from each other. Maxima of value $L$ are approximately $250 \mathrm{~dB}$ higher in the case when the PD was induced in the winding. This may be due to the fact that the pathway was $0.2 \mathrm{~m}$ shorter than in the case when the PD was induced in the core and due to different physical properties of transformer elements.

\section{Summary}

Acoustic wave propagation inside a power transformer was analyzed in this paper. The acoustic wave was initiated by partial discharges, which were simulated in the core and in the winding inside the oil filled transformer. The wave equation was calculated numerically using the finite element method supplied by the COMSOL Multiphysics application. The transient distribution of the acoustic pressure was analyzed at different Cartesian coordinates at the transformer surface. Achieved results indicate different space and time distributions of the acoustic pressure if the PD induced at diverse places. In general, a greater pressure gradient was observed in domains with higher speed of sound while the amplitude was highest at the vicinity of the induced PD. Future work should include more sophisticated model of the transformer as well as it should consider wave attenuation in the different media (core, windings, bases, oil). Also fluid dynamics and the impact of other physical phenomena are to be considered. 


\section{References}

[1] F. Ozaki, R. Akumu, A.O. Ihori, H. Fujii, M. Arii, in: Proc. 2002 IEEE Int. Symp. on Electrical Insulation Materials, Himeji (Japan), 2001, p. 880.

[2] R. Akumu, O. Masai, H. Adachi, F. Ozaki, R. Ihori, H. Fujii, M. Arii, Jpn. J. Appl. Phys. 42, 3686 (2003).

[3] R. Akumu, A.O. Kawaguchi, N. Ozaki, R. Ihori, H. Fujii, M. Arii, in: Proc. 2001 IEEE Int. Symp. on Electrical Insulation Materials, Himeji (Japan), 2001, p. 583.

[4] R. Akumu, A.O. Adachi, F. Kawaguchi, N. Ozaki, R. Ihori, H. Fujii, M. Arii, in: IEEE Int. Symp. on Electrical Insulation, Boston (MA, USA), 2002, p. 183.

[5] R. Rausch, M. Kaltenbacher, M. Landes, H. Lerch, Int. J. Comput. Math. Electr. Electron. Eng. 20, 636 (2001).

[6] G. Hettiwatte, S.N. Crossley, P.A. Wang, Z.D. Darwin, A. Edwards, in: IEEE Power Engineering Society Winter Meeting, 2002, Vol. 2, p. 1394.
[7] J.M. Ashraf, S.A. Stewart, B.G. Chengke, Z. Hepburn, D. Jahabar, in: Proc. Conference Record of the IEEE Int. Conf. on High Voltage Engineering and Application, Chongqing (China), 2008, p. 577.

[8] T. Boczar, D. Zmarzly, IEEE Trans. Diel. Electr. Insulat. 11, 433 (2004).

[9] F. Witos, Z. Gacek, Z. Opilski, Acta Phys. Pol. A 114, A-249 (2008).

[10] T. Boczar, S. Borucki, A. Cichon, M. Lorenc, Acta Phys. Pol. A 114, A-21 (2008).

[11] F. Witos, Z. Gacek, P. Paduch, Mol. Quant. Acoust. 28, 271 (2007).

[12] T. Boczar, M. Szmechta, D. Zmarzły, Mol. Quant. Acoust. 28, 39 (2007).

[13] A. Szpakowski, C. Tyszkiewicz, T. Pustelny, Acta Phys. Pol. A 114, A-237 (2008).

[14] T. Boczar, S. Borucki, A. Cichon, Mol. Quant. Acoust. 28, 13 (2007) 